\title{
Reconsidering the modularity of social cognition in the human brain
}

\author{
Alvaro M. Dias* \\ Neurosciência e Comportamento, Instituto de Psicologia, Universidade de São Paulo, São Paulo, Brazil \\ ${ }^{*}$ Correspondence: alvaromd@usp.br
}

The concept of modularity is used to express the division of the computational resources of the mind into several mental expertise systems. This idea gained major importance within the emerging field of social neuroscience (Cacioppo et al., 2000), as it brought together three lines of studies that up to that point were not so easily combined: studies on the neurobiological correlates of complex traits, studies on the evolutionary basis of these correlates, and studies on social behavior (for a classical example: Fiddick et al., 2000).

By establishing the importance of a limited number of principles inherent to the brain, this concept of modularity represents a reaction to the relativist tradition that dominated studies of social phenomena during twentieth century, which strongly emphasized cross-cultural differences in social behavior and its irreducibility to biological frameworks (for a discussion: Hirschfeld and Gelman, 1994). Conversely, the perspective that group cohesion relies on specific, semi-automatic types of cognition, which embody unique responses to selective pressures that lead to the differentiation of brain circuits specialized for those demands (Cosmides, 1989) greatly expands the field of cognitive sciences, and that is one of the main reasons why it became so prominent.

Among the social cognitive processes that define this expertise system, the most prominent are Theory-of-Mind (BaronCohen, 1997), sensitivity to fairness (Barkow et al., 1992), and in-group/outgroup differentiation (Ohman, 2005). It is reasonable to assume that sensitivity to fairness and in-group/out-group differentiation are especially related: not only do they exert highly complementary functions, but they also represent complementary types of modular learning, related to the propensity to generate long-lasting reactions to unfair in-group members and to out-group members paired with aversive stimuli.

One of the most prominent experiments on in-group/out-group differentiation demonstrated that members of a racial group other than one's own were more readily and lastingly associated to aversive stimuli in the classic conditioning paradigm (Olsson et al., 2005). The authors hypothesized that this trend was related to an evolutionary predisposition to fear out-group members, in accordance with the argument that race bias activates subcortical systems that are similar to the ones activated by fear learning.

In relation to fairness, one of the most prominent perspectives establishes that most people tend to reject low offers in the Ultimatum Game, despite the fact that this behavior stands against the maximization of their profits, whereas chimpanzees tend to be 'rational maximizers' (Jensen et al., 2007). This perspective is often related to findings indicating the role of ventromedial prefrontal cortex in regulating utilitarian behavior in human subjects (Koenigs, et al., 2007), to the same extent that it is related to studies arguing that source memory of the faces of cheaters (Mealey et al., 1996; Buchner et al., 2009) and unfair participants is enhanced (Oda, 1997).

However, two very recent studies contest the conclusion that these findings lead to the endorsement of a social expertise system. In the first one, Tiago Maia (2009) revealed that the superior out-group conditioning effects that were found in the race experiment could be explained solely based on the perspectives that lower exposure to members of the other race led to increased learning rates and that prior exposure to members of the other race was different for whites and blacks. He reached this conclusion by applying a statistical method (the Kalman Filter), commonly used to analyze phenomena that change continuously over time, to a computational simulation of the experiment that included all its parameters and found that the participants of both races behaved in the way expected under a normative statistical model of learning that did not include any weight to a priori/evolutionary preparedness to fear out-group members. According to the author, this analysis explains a subtle and yet important feature of the original American experiment: outgroup conditioning was slightly higher for whites than for blacks, due to the fact that blacks have a mean exposure to whites that is higher than the opposite.

In the second and more remarkable study, Chang and Sanfey (2009) revealed that the amount of money offered in the Ultimatum Game did not correlate to the chances of being remembered by an opponent, after several game sessions with different subjects, as would be expected if there was an evolutionary tendency to remember coparticipants, based on their fairness. On the contrary, the authors found that selective memory for faces was actually correlated with the intensity to which an opponent violated the subject's expectations in any direction. Making an analogy with Maia's experiment, one would say that it is not necessary to attribute differential weights to the different types of outcomes of the social interaction (fair/unfair) in order to model the way that the participants of this experiment remember the ones with whom they interacted, since the learning rates are solely defined by expectation. This experiment also involved neuroimaging, which led the authors to add that there is no particular neural signature for either fair or unfair partners encountered previously (Chang and Sanfey, 2009; p. 12), therefore extending the social cognitive findings to the neurobiological bedrock.

Together these experiments inspire a note of caution in regard to radically modular views of human social cognition, as they expose some potential weaknesses at the heart of the concept of social expertise and its use to explain complex behavioral findings. It is not the case to say that they deny the existence of a social brain module (clearly, this denial is not pursued by the authors), but it remains a fact that they present evidence that the interaction between cross-domain 
faculties and expertise cognition might not be as simple as it was once conceived. Specifically, this could mean that the perspective that specialized systems would 'pre-empt' more general ones (Fiddick et al., 2000) might be challenged by more complex statistical arrangements, in which less specialized principles received more attention.

\section{REFERENCES}

Barkow, J., Cosmides, L., and Tooby, J. (1992). The Adapted Mind: Evolutionary Psychology and the Generation of Culture. New York, Oxford University Press.

Baron-Cohen, S. (1997). Mindblindness: An Essay on Autism and Theory of Mind. Cambridge, The MIT Press.

Buchner, A., Bell, R., Mehl, B., and Musch, J. (2009). No enhanced recognition memory, but better source memory for faces of cheaters. Evol. Hum. Behav. 30, 212-224.

Cacioppo, J. T., Berntson, G. G., Sheridan, J. F., and McClintock, M. K. (2000). Multilevel integrative analyses of human behavior: social neuroscience and the complementing nature of social and biological approaches. Psychol. Bull. 126, 829-843.

Chang, L. J., and Sanfey, A. G. (2009). Unforgettable ultimatums? Expectation violations promote enhanced social memory following economic bargaining. Front. Behav. Neurosci. 3. doi:10.3389/neuro.08.036.2009

Cosmides, L. (1989). The logic of social exchange: has natural selection shaped how humans reason? Studies with the Wason selection task. Cognition 31, 187-276.

Fiddick, L., Cosmides, L., and Tooby, J. (2000). No interpretation without representation: the role of domainspecific representations and inferences in the Wason selection task. Cognition 77, 1-79.

Hirschfeld, L., and Gelman, S. (1994). Mapping the Mind: Domain Specificity in Cognition and Culture. Cambridge, Cambridge University Press.

Jensen, K., Call, J., and Tomasello, M. (2007).Chimpanzees are rational maximizers in an ultimatum game. Science 318, 107-109.

Koenigs, M., Young, L., Adolphs, R., Tranel, D., Cushman, F., Hauser, M., and Damasio, A. (2007). Damage to the prefrontal cortex increases utilitarian moral judgements. Nature 446, 908-911.
Maia, T. V. (2009). Fear conditioning and social groups: statistics, not genetics. Cogn. Sci. 33, 1232-1251.

Mealey, L., Daood, C., and Krage, M. (1996). Enhanced memory for faces of cheaters. Ethol. Sociobiol. 17, 119-128.

Oda, R. (1997). Biased face recognition in the Prisoner's Dilemma Game. Evol. Hum. Behav. 18, 309-315.

Ohman, A. (2005). Psychology: conditioned fear of a face: a prelude to ethnic enmity? Science 309, 711-713.

Olsson, A., Ebert, J. P., Banaji, M. R., and Phelps, E. A. (2005). The role of social groups in the persistence of learned fear. Science 309, 785-787.

Received: 25 October 2009; published online: 09 December 2009

Citation: Front. Behav. Neurosci. (2009) 3:55. doi: 10.3389/neuro.08.055.2009

Copyright (c)2009 Dias. This is an open-access article subject to an exclusive license agreement between the authors and the Frontiers Research Foundation, which permits unrestricted use, distribution, and reproduction in any medium, provided the original authors and source are credited. 